\title{
Psychological Stress, Self-Compassion and Self-Punishment among Tension-Type Headache and Healthy University Students (A Comparative Study)
}

\author{
Sawzan S. Basyouni ${ }^{1}$ \\ ${ }^{1}$ Associate Professor of Mental Health and Psychological Counseling, College of Education, Umm al-Qura \\ University \\ Correspondence: Sawzan S. Basyouni, Associate Professor of Mental Health and Psychological Counseling, \\ College of Education, Umm al-Qura University, Saudi Arabia. E-mail: ssbasuoni@uqu.edu.sa
}

Received: September 11, 2019

Accepted: October 7, 2019

Online Published: October 10, 2019

doi:10.5539/ijps.v11n4p53

URL: https://doi.org/10.5539/ijps.v11n4p53

\begin{abstract}
Headache is one of the most common complaints among university students due to the lifestyle they lead and the academic and social burdens they have. The present study aims to identify the differences between Tension-Type Headache (TTH) and healthy university students regarding psychological stress, self-compassion, and self-punishment. It covered a sample of tension-type headache and healthy students visiting the medical center and university clinic at Umm Al-Qura University and University of Jeddah numbered (235) and aged (18-40) years. To achieve the study objectives, an analytical descriptive approach was adopted. A TTH scale and a psychological stress scale, prepared by the author, in addition to a self-compassion scale and a self-punishment scale were applied. Results revealed that there were statistically significant differences in the means of TTH and healthy students at the level of (0.01) on the scales of psychological stress, self-compassion, and self-punishment in favor of students with TTH. The study recommends developing consulting programs to reduce psychological stress, self-compassion, self-punishment, and their somatic symptoms among university students.
\end{abstract}

Keywords: psychological stress, self-compassion, self-punishment, tension-type headache

Contribution/Originality: This study contributes to the existing literature that deals with psychological disorders in relation to their somatic symptoms among university students. No previous study has tackled psychological stress, self-compassion, and self-punishment in relevance to TTH.

\section{Introduction}

Rapid and complicated changes in life, social, and economic styles have resulted in the prevalence of chronic and persistent diseases such as cardiovascular diseases, TTH, and diabetes affecting human activity and psychosomatic health. Unsurprisingly, psychological factors, emotional stress and every-day life constrains are the main causes of the prevalence of such diseases.

Headache is a top complaint not only among patients with differing diseases, but also among healthy people. It is a common symptom of different somatic and psychological diseases. Statistics report that headache highly prevails among (90\%) of the world population whereas males and females are rated $(93 \%)$ and $(99 \%)$, respectively (Rasmussen, 2001).

TTH is the most common form of headache representing $(90 \%)$ of primary headache prevails in $(78 \%)$ of the patients with headache (63\% males and $86 \%$ females) (Torelli et al., 2008). It is associated with muscle tension due to air currents, poor postures, or brain injuries, in addition to emotional states, e.g. shocks, hatred, or stress (Hanafy, 1992).

Patients with TTH complain of persistent and indefinite pain in the head, commonly in the back of the neck or in the forehead. It can be recurrent and becomes intense at night. It requires a certain medication containing antianxiety agents and antidepressants along with psychotherapy (Awad, 2002).

In the same context, Farghaly (1995) argues that TTH is a model suitable for investigating the relationship between psychological factors and somatic symptoms. It is a common psychosomatic disorder caused by many psychological illnesses, such as anxiety, depression, stress, and neurosis resulting in many somatic symptoms. 
According to Shoqer (2002), general health plays a vital role in mental health because the body-mind relationship is interactive, i.e. somatic diseases may cause psychological disorders or distress, low academic achievement, and psychological and social maladjustment.

Nowadays, people are prone to psychological stress resulting from the daily life's complications and problems that require immediate solutions. Consequently, they tends more to self-punishment and self-compassion causing many psychosomatic diseases and TTH.

There are more than (60) concepts related to the self, including for instance self-concept, self-esteem, and self-acceptance. The concepts of self-compassion and self-consciousness have appeared in the early $21^{\text {st }}$ century. Self-Compassion is a relatively new concept in Psychology. It is appositive attitude towards the self and entails being warm towards oneself when encountering pain and personal shortcomings, rather than self-judgment (Abdurrahman et al., 2015; Alasemy, 2014; Mansour, 2016).

Neff (2003) has coined the concept of self-compassion and developed a scale to measure it. It includes a basic domain of self-construction, an important trait of the positive personality, and a barrier to the negative implications of stressful life events, especially when a person encounters failure or personal incompetence in resolving personal problems (Abdurrahman et al., 2015; Almenshawy, 2016). Moreover, Neff and McGehee (2010) report that self-compassionates differ from non-compassionate ones in terms of the personality traits. They show higher levels of understanding, empathy, and resilience rather than severe self-judgment. They are also more open to experience and more rational in addressing negative experiences (Alasemy, 2014).

Negative emotions, e.g. self-abasement and dissatisfaction may result from a cognitive structure, beliefs, and prior thinking. Negative and distorted thinking produces negative emotion and behavior that affect self-adjustment (Ashareef, 2006). That is, self-abasement, which results from fear, may entail low self-esteem.

Self-abasement is associated with shame and low self-esteem, tendency to loneliness, incompetence, humiliation, and self-loathing. It is a personal reflection of the feeling of the self. It may be a defense mechanism against anxiety, fear, and anger (Saema, 2018).

It may be noted that patients with TTH may be stressful, hard workers, achievement and mastery seekers, and highly self-critical (Alkhaled, 2005; Shoqer, 2002). They may find it hard to express their feelings and have limited imagination, infrequent dreams, and absorption of the details of external events. As a result, they build limited social relations, increased psychological stress, and inability to enjoy life (Dahmash, 2017).

Therefore, it is important to study TTH and its prevalence. There are only few studies in the field. The present study aims to identify the differences between TTH and healthy university students regarding psychological stress, self-compassion, and self-punishment at Umm Al-Qura University and University of Jeddah.

\subsection{Statement of the Problem}

Ghanim (2009) and Shoqer (2002) report that most patients with TTH do not have physical illnesses. However, they encounter increasing health and psychological problems, e.g. a permanent pain in the head, tightness of the neck muscles, and pain behind the eye. This pain does not continue to worsen because of daily physical activity (Saudi Ophthalmological Society, 2017). The author believes that headache may be caused by psychological and social stress, anxiety, fear, tension and continuous excitement and the inability to confront them.

TTH greatly affects the psychological and social life resulting in social maladjustment and many psychological disorders, e.g. stress, self-compassion, and self-punishment. This was reported by Beghi et al. (2007), Fielding et al. (2016), Mongini et al. (2006), Torelli et al. (2008), and Saema (2018) regarding the correlation between TTH and psychological disorders, especially stress. Torelli et al. (2008) argue that (63\%) of the males and (86\%) of the females are rated patients with TTH.

Recent studies have investigated self-compassion as an important and positive variable in the individual's personality. It covers an important aspect of the psychological structure, a feature of the positive personality, and a resistant variable to the negative implications for stressful life events. This could happen when a person experiences failure or inadequacy in resolving life problems (Almenshawy, 2016). Alanzey (2007) highlights the inventory of somatic symptoms that university students are prone to and its different degrees, especially persistent headache. In addition, he attributes the prevalence of this disorder to internal and external psychological stress.

Söderlund and Lagerlöf (2016) and Fielding et al. (2016) recommend conducting further studies on adolescents with TTH to define and address its psychological causes using different behavioral styles. There are only few Arabic studies in the field and the theoretical framework is limited. Therefore, the study raises the following 
major question: Are there statistically significant differences between TTH and healthy university students regarding psychological stress, self-compassion, and self-punishment?

It is further divided into the following minor ones:

1. Does the degree of psychological stress differ according to health status (TTH- healthy students)?

2. Does the degree of self-compassion differ according to health status (TTH- healthy students)?

3. Does the degree of self-punishment differ according to health status (TTH- healthy students)?

\subsection{Significance}

The present study is significant because it highlights the most common symptoms of pain. The Diagnostic and Statistical Manual of Mental Disorders- Fourth Edition (DSM-IV) reports that stress is one of the psychological factors that cause somatic symptoms, i.e. TTH, which prevails in more than half of the patients seeking treatment. It is the common symptom of somatic and psychological diseases (Hanafy, 1992) and it prevails in (78\%) of the patients (Torelli et al., 2008). In this study, we highlight some personality variables, including psychological stress, self-compassion, and self-punishment among patients with TTH. The results may help those in charge of the treatment understand the personality of the patient to reduce tension and treat headache. The study adds important tools to the psychological Arab library, such as developing a diagnostic scale of TTH as well as psychological stress scales. It may contribute to developing counseling programs for improving self-image, self-adjustment, and dealing with life stress among people who suffer from TTH's symptoms.

\subsection{Definition of Terms}

Tension-Type Headache (TTH): It is a psychological-based headache that results from different stressful experiences. It is accompanied by many physiological pains in the head, skull, and back of the neck, along with severe tightness on the head. Therefore, the patient usually experiences stress, tension, depression, and fear that spoil her/ his life and causes psychological and social maladjustment (Shoqer, 2003).

The author defines it as a severe headache due to the psychological factors experienced by the patient in the form of somatic symptoms that make him/her unable to enjoy life, including severe pain in the head and neck and blurred vision. Therefore, the patient usually experiences tension, permanent distress, and inability to adjust. It is procedurally defined as the score that the participant receives on the scale of TTH (developed by the author).

Psychological stress: It is a set of the internal and external stressful factors that results in the inability to respond adequately to the event. It is accompanied by emotional and physiological disorders that affect the other personality traits (Shoqer, 2002). It is defined procedurally as the score that the participant receives on the scale of psychological stress and its coping methods. The author defines stress as painful, unpleasant, and anxious events that cause the inability to enjoy life and the occurrence of many psychological and personal disorders, fatigue, recurrent pain, and inability to confront.

Self-compassion: Neff (2003) defines self-compassion as a positive self-attitude in hard or failing situations. It involves being touched by and open to one's own suffering, not severely criticizing it. It also involves understanding this suffering as a common one and healing oneself with kindness and openness (Alobaedy, 2017). Procedurally, it is defined as the score that the participant receives on the self-compassion scale and its domains, i.e. self-kindness vs. self-judgment, common humanity vs. isolation, and mindfulness vs. over-identification.

Self-punishment: Shoqer (2006) defines it as a set of abnormal and strange behaviors through which the person harms or punishes himself/ herself on periods and in different contexts. It takes the form of punishment or intended damage towards parts of the body and expresses anger, sadness, and rage in an attempt to torture the self and please the others. It may be chronic feeling of neglect, inferiority, or humiliation resulting in a distorted self-image. Procedurally, it is defined as the score that the participant receives on the scale of self-punishment and its domains, i.e. self-injury, emotional-abuse, self-neglect, and self-deprivation (prepared by Shoqer, 2006).

\subsection{Theoretical Framework}

\section{Tension-Type Headache}

Causes:

1. Somatic causes:

- TTH is caused by tightness around the muscles of the face, neck, and scalp causing expansion and contraction of the blood vessels, which makes the headache worse.

- Injuries of the backbone. 
- $\quad$ Poor posture (Donaghy, 2001; Murtagh, 1995)

- Contraction of the muscles linking the back and front parts of the head and the neck.

- High concentrations of some chemicals in the blood, e.g. adrenaline, which cause muscle tension (Farghaly, 1995).

Recent studies report that changes in the level of serotonin and endorphin in the brain play a role in the occurrence of TTH (Aaseth et al., 2009).

2. Psychological causes:

Many authors such as Alesawey (2002), Kaufman (1990), Murtagh (1995), and Shama (1997) discussed the psychological causes of TTH, including failure, deep frustration due to over ambition, fanaticism, high sensitivity, self-judgment, tension, poor psychological state, anxiety, excessive fear, aggression, anger, unhealthy food habits, depression, hysteria, and the inability to handle psychological and social stress. Kaufman (1990) believes that excitement, tension, and stress are responsible for (90\%) of TTH. Moreover, Belqassem (1992) argues that the acquired psychological causes are responsible for TTH. Elsherbeny et al. (2018) suggest that TTH may result from excessive stress, anxiety, repressed anger, impatience, competition, over ambition, disorganization, and excessive work.

Therefore, the author argues that TTH occurs because of professional, social, and psychological causes, as well as emotional stress and genetic causes (oligoencephaly), personality type, beliefs and daily practices, and severity and continuity of fatigue.

3. Environmental and social causes:

Eye strain that results from excessive paperwork, reading for long hours, continuous work, long-term study, writing, long-term concentration, earnestness, and perfectionism (Murtagh, 1995). Social and environmental problems and family disputes may cause TTH among highly sensitive persons, in addition to long exposure to severe disturbance or darkness, high peer-competition, poverty, and low economic status of the family (Alesawey, 2002; Farghaly, 1995; Izmailova et al., 2002).

Symptoms:

1. Somatic symptoms:

TTH is often accompanied by many somatic complaints, including tight head pain, especially in the head sides and forehead. The patient sometimes has chronic pain in the neck or back part of the head, along with expansion and contraction of the muscles (Bannister, 1992; Donaghy, 2001; Kleiner-Fisman, 2002; Shoqer, 2009; Waldie and Poulton, 2002).

\section{Psychological symptoms:}

Patients with TTH may suffer from anxiety, depression, cruelty, and tendency to defensive tricks as a psychological reaction, as well as psychological and emotional stress, thinking disorders, difficulty of concentration, and psychological disorders (Juang et al., 2000; Stronks et al., 1999).

\section{Social symptoms:}

Many studies, e.g. Carlsson et al. (1996), Dubojska et al. (1998), Omdal et al. (2001), Shoqer (2005), and Stronks et al. (1999), agree that patients with TTH tend to utilize defensive tricks instead of seeking social or external support on encountering problems. They demonstrate introversion, social isolation, psychosocial stress, anxiety, and social withdrawal. Furthermore, the patient encounters excessive truancy, lack of interest compared to peers, interest in clothing cleanliness, compulsive accuracy in planning and implementing issues, high sensitivity to criticism, judging others, over ambition, and forcing the self to achieve high objectives.

\section{Psychological stress}

Causes:

Shoqer (2002) argues that psychological stress is caused by:

- $\quad$ Psychological (emotional) problems, such as anger, depression, and apathy;

- $\quad$ Economic problems;

- Family problems;

- $\quad$ Social stress that includes poor interpersonal relations and difficulty of making friends; 
- Physiological problems, e.g. headache, hypertension, gastroparesis, heart palpitations, nausea, dizziness, and tremor;

- Personal problems, e.g. withdrawal, resistance, low self-esteem, low level of ambition, and intellectual hardness; and

- $\quad$ Academic problems, e.g. difficulty of interacting with colleagues and teachers, poor achievement, low concentration, inability to do homework, and examination failure.

Effects on the mental health:

Many studies have investigated the effects of stress on the emotional, cognitive, and behavioral aspects. They include excessive stress, inability to relax, chronic anxiety, high rates of obsessive-compulsive disorder, frequent complaint from continuous fatigue without any physical cause, change in personality traits, depression, irritation, losing hope, guilty, pessimism, and self-defeating (Askar, 2000; Fontana, 1993; Ismael, 2004).

Coping methods

Lazarus et al. (1982) report that confronting stress includes two processes:

- Problem-based coping that takes many forms, including preparation for the situation, resilience, and avoidance.

- Emotion-based coping that relies on:

a. Physiological techniques, i.e. using some sedatives, painkillers, and relaxation techniques.

b. Internal psychological techniques, including mental defensive tricks.

The author argues that psychological stress is involuntary painful responses to emotional disorders in the form of somatic symptoms. The person experiences imbalance, fear, ungiving and life spoil due to anxiety and tension. To avoid psychological, somatic, and behavioral disorders and diseases, the person should control the way of confronting and managing stress. S/ he should learns how to control the self, attitudes, and emotions to define the origin of stress, confront, and change the coping strategy. This is reflected in her/his life and thinking style limiting the effect of bad emotions and psychological stress.

\section{Self-compassion}

Aspects and components

Almenshawy (2016) reports that self-compassion has two aspects, i.e. the positive aspect that includes self-kindness, common humanity, and mindfulness and the negative aspect that includes self-judgment, isolation, and over-identification. It comprises:

1. Self-kindness vs. self-judgment: It is a state of self-understanding in the situations of failure, frustration, and suffering instead of hard judgments, as well as showing a positive self-effect instead of criticism. It also includes understanding and emotional warmth towards the self, especially in suffering, failure, and frustrating stressful events. This helps suffered or failed individuals to manage and handle stress objectively.

2. Common humanity vs. isolation: It involves recognizing that suffering and personal inadequacy is part of the shared human experience and achieving a state of balance between the self and others. It is a participatory case that allows to see personal experiences and others' sufferings as integral. That person believes that it is part of the common suffering and a common human experience.

3. Mindfulness vs. over-identification: It is the balanced awareness that prevents over-identification. Mindfulness helps people change their thinking style and handling experience, especially the painful and stressful one that cause failure and frustration through reflection and deep breath. Instead of surrender and hurting when encountering failure, a person should cope (Abdurrahman et al., 2015; Alasemy, 2014; Alobaedy, 2017; Olwan, 2016).

Traits of the self-compassionate

In painful situations, self-compassionate persons demonstrate different personal traits. They are more flexible, open to experience, and rational in addressing negative experience. Self-compassion relates to happiness, optimism, and maturity. When those persons undergo painful experience or failure, they think of themselves in an understanding, coherence, and passionate way instead of over-judgement or self- abasement.

The author believes that self-compassion is an internal negative or positive emotional feeling towards the self when having stressful or painful experience. The person sees his/her life apart from suffering and perceives the negative ideas that cause uneasiness and surrender. 


\section{Self-punishment}

It is one of the basic concepts of mental health, which interferes with other relative psychological concepts, e.g. guilty, aggressiveness, and hostility. Many psychologists neglect the interest and procedural definition of the term. However, Alkhateeb (1993) suggested many terms:

- $\quad$ Self-abuse

- $\quad$ Self-mutilation

- Self-damage

- $\quad$ Self-punishment

Shoqer (2006) reports that the modern classifications of self-punishment have focused on the physical aspect (physical self), mental self, and emotional self. The author argues that self-punishment is a psychological pain due to events and disorders in childhood or any other life stage that have affected personality and self-concept. They may have many psychosomatic symptoms. Thus, they affect self-concept and cause guilty and emotional or social immaturity even in the simplest situations.

\section{Literature Review}

Because of their importance, TTH, psychological stress, self-compassion, and self-punishment are the subject of many studies. The author reviewed the literature on TTH, psychological stress, self-compassion, and self-punishment and their relationship to some variables related to and affecting mental health. This review could help identify and measure them and define their correlation to some variables.

\section{TTH and its relation to some variables}

Venable et al. (2001) explored the role of anger in headaches and examined its relationship to anxiety, depression, and daily life stressors. The study covered 65 young adult women with recurrent headaches. Results highlighted the serious relationship between repressed anger and depression, as well as repressed anger and anxiety on adult women with headache. Moreover, adult women with headache had a general state of sadness and manifested symptoms indicating anxiety and depression.

Sarioglu et al. (2003) aimed to define the criteria of TTH among children (a clinical evaluation). The study covered the criteria, symptoms, features, causes, factors, and psychological and social evaluation of TTH in the International Headache Society according to DSM-IV. The study covered a sample of children with TTH. Results revealed that pain increases with the increase of stress and fatigue among more than half of the patients, but it decreases with relaxation and massage among (43.7\%) of the patients. Difficulty in adaptation at school and family quarrels were the most significant headache triggers.

Leeuw et al. (2005) investigated the prevalence of traumatic stressors and post-traumatic stress disorder (PTSD) symptoms in headache patients. The study applied PTSD checklist to (80) patients with migraine and TTH. Results reported that $(64 \%)$ of the patients suffered from stress and fatigue. There was a relationship between exposure to traumatic events and recurrent headache.

Perozzo et al. (2005) examined the relationship between anger and emotional distress in migraine and TTH patients. The study covered (201) headache patients divided into 5 groups: (1) migraine, (2) episodic TTH, (3) chronic TTH, (4) migraine associated with episodic TTH, and (5) migraine associated with chronic TTH. Moreover, a group of (45) healthy subjects served as controls. Results showed that chronic TTH patients present a significant impairment of anger control. They suggested a correlation between anger, fear, depression, and TTH.

Wöber et al. (2006) aimed to define the trigger factors of migraine and TTH and to compare the patients' personal experience with their theoretical knowledge among (120) participants. Results illustrated that the external factors greatly affected headache. Weather, fatigue and psychological stress, and menstruation were rated $(82.5 \%),(66.7 \%)$, and $(51.4 \%)$, respectively.

Torelli et al. (2008) aimed to explore the psychological factors associated with TTH. It covered a sample of the patients with TTH. Results reported a correlation between TTH and psychological disorders, especially anxiety and depression. Moreover, patients with TTH might have difficulty in expressing their emotions. The psychological factors and emotional disturbances were indicated as risk factors for TTH and stress and mental tension are the most common factors that cause it.

Simić et al. (2008) aimed to identify the quality of life among patients suffering from TTH. The study also explored the effect of TTH on social and family life and production. It adopted Qualite de Vie et Migraine 
(QVM) questionnaire. Results revealed that there were significant differences in the quality of life between the patients with TTH and the control group. The patients showed disorder and tension in the quality of life that negatively affect their social and practical life.

Kumar et al. (2016) aimed to examine the prevalence of TTH among patients of depressive disorder. The study covered a sample of (264) patients; single or married men and women. Results revealed that $(21.2 \%)$ of the participants experienced TTH, especially married women.

Carasco and Kröner-Herwig (2016) aimed to examine the psychological variables as potential predictors of headache remission among children and adolescents. The study covered a sample of (509) participants aged 9 to 15 years who suffered from weekly headaches. Results revealed that highly dysfunctional coping strategies reduced the relative probability of headache remission. All other selected psychological variables reached no significance. Successful coping with stress in general contributed to remission of pediatric headache after 2 years in children aged between 9 and 15 years. Psychological characteristics in general had only small predictive value.

Fielding et al. (2016) aimed to examine the incidence of headache symptoms chronic with migraine and TTH in children with anxiety disorders. The study covered a sample of (27) parents of children with anxiety disorder and (36) parents of children with no anxiety disorder. Results revealed that the children with anxiety disorder were higher in the symptoms of migraine and TTH. The girls with anxiety disorder and children with detachment anxiety were higher in the percentage of headache. Moreover, the results asserted that anxiety and TTH overlap among children.

Söderlund and Lagerlöf (2016) aimed to describe and evaluate an individually tailored behavioral medicine treatment in physical therapy for TTH and to develop functional behavioral analysis for TTH. The sample covered (3) patients undergoing physical therapy. Results showed that headache decreased for (1) patient, while self-efficacy regarding the treatment of headache significantly increased for all participants. Therefore, they were satisfied with the activities with the family and friends.

Ruby and Ibn Hany (2018) aimed to investigate the relationship between TTH and mental health. The study also aimed to identify the differences in the mean scores of secondary school teachers on the scales of TTH and mental health based on gender, specialization, and seniority. It covered a sample of (140) participants. Results revealed that there was a statistically significant negative correlation between TTH and mental health. While there were no differences in the degree of TTH due to gender and seniority, there were differences due to specialization.

\subsection{TTH and Its Relation to Psychological Stress}

Assahley, A. (2010) aimed to define the styles of confronting psychological stress among patients with depression and anxiety. The study covered a sample of (210) participants distributed to two groups; (140) patients and (70) healthy. It utilized the scale of stress confrontation developed by Alanbary (1990). Results revealed that there was a statistically significant difference between the patients and the healthy. Moreover, the patients tended more to using the negative styles of stress confrontation.

Qlandar and Abbas (2017) aimed to investigate emotional suppression and psychological stress, as well as the strength and direction of their relation among university students. To achieve its objective, it selected (400) university students in the scientific and humanity specializations; (160) males and (240) females. It adopted the 24-item scale of emotional suppression (Taleb, 2013) and the 82-item scale of psychological stress (Alqaessy, 2008). After defining the psychometric characteristics, the scale comprised (22) items of emotional suppression and (82) items of psychological stress. Results revealed that the university students had a lower level of emotional suppression and a higher level of psychological stress than the standard ones. There was a positive direct relationship between emotional suppression and psychological stress; the higher psychological stress was, the higher level of emotional suppression the students had. Furthermore, there were no differences in the relationship between psychological stress and emotional suppression according to gender (males and females) and stage (first and fourth). Based on the results, the author made some recommendations and suggestions.

\subsection{Self-Compassion and Its Relation to Some Variables}

Neff, Kirkpatrick, and Rude (2007) aimed to examine the relation of self-compassion to some positive personality characteristics. The study covered a sample of (177) university students. It adopted the self-compassion scale, 5-item self-kindness scale, and mindfulness scale. Results revealed that there was a positive correlation between self-compassion and mindfulness, openness to experience, and common humanity. There was a negative relation to neurosis. 
Crocker and Canevello (2008) aimed to investigate the relationship between self-compassion and social support among (199) university students. Results revealed that self-compassionate tend to help others and to create strong relationships with them. This, in turn, is reflected on them. Moreover, they do not seek to achieve personal interests. They help others and enjoy clarity, communication, and closeness to others. They are less conflictive.

Ying (2009) aimed to investigate self-compassion to competence and mental health among social work student. The study covered (65) students. It utilized the self-compassion scale and the scale of mental health and psychological resilience. Results revealed that there was a significant relationship between mindfulness, the domains of the self-compassion scale, and other variables.

Alasemy (2014) aimed to define the relationship between self-compassion and personality traits based on the list of five personality traits and to identify the differences in self-compassion due to gender and specialization. Results revealed that there was a positive relationship between the positive aspects of self-compassion (self-kindness, common humanity, and mindfulness) and personality traits (openness, open to experience, acceptability, and consciousness). There was a negative relationship between self-judgment, isolation, over-identification, and neuroticism. Moreover, the females were more self-compassionate than the males and the students of the literary colleges are more self-compassionate than those of the scientific colleges. There was a statistically significant correlation between gender, specialization, and self-compassion.

Olwan (2016) aimed to identify the level and correlation of self-compassion and guilty. The study covered a sample of (53) juvenile offenders at Social Observation House in Abha. It concluded that there is a low level of self-compassion and a high level of guilty among participants. There was a statistically significant correlation between self-compassion and guilty. Furthermore, the aspects of self-compassion help predict guilty.

\subsection{Self-Punishment and Its Relation to Other Variables}

Saleh (2002) aimed to explore the relationship between self-punishment and psychosomatic diseases. The study covered a sample of (75) patients aged 35-64 years with psychosomatic disorders, e.g. TTH and hypertension. Results revealed that the freelancers had a higher level of self-punishment than the employees. The divorced had a higher level of self-punishment than the married and single. In addition, self-punishment is a major trigger of psychosomatic diseases.

Alaaeldeen (2003) aimed to identify the relationship between guilty and self-punishment among the students of the Hashemite University. The study was covered a sample of the students at the Faculty of Educational Sciences. It revealed that there was a correlation between guilty and self-punishment. Moreover, self-punishment might be due to guilty.

Ashaboun (2012) aimed to explore the differences among adolescents in guilty and self-punishment according to gender. The study covered a sample of (655) students. Results revealed that there was a correlation between guilty and self-punishment among the participants. There were no differences between the males and females in self-punishment.

Almomney and Shawashra (2013) explored the relationship between self-punishment and irrational beliefs among the residents of the Jordanian Reformation and Qualification Centers. The study covered a sample of (57) residents with self-punishment and (58) normal residents. Results revealed that the residents with self-punishment had a higher level of irrationality. As-Saeedy (2017) explored the relationship between self-punishment and aggression among (60) adolescents based on their responses to the self-punishment scale and the aggressive behavior scale. Results showed that there were statistically significant differences in self-punishment due to gender.

\subsection{Commentary}

Many studies investigated TTH and its causes, such as Kumar et al. (2016), Leeuw et al. (2005), Mongini et al. (2006), Perozzo et al. (2005), and Simić et al. (2008). They reported that it is one of the most common psychological disorders. It is significantly related to the psychosomatic diseases, e.g. psychological stress, anxiety, and depression. In addition, the samples varied from university students and children, married and single, to different environments, such as Fielding et al. (2016) and Kumar et al. (2016).

Some studies addressed self-compassionate and its triggers, as well as its relationship to guilty, social support, and personality traits, e.g. Alasemy (2014), Alobaedy (2017), Crocker and Canevello (2008), and Olwan (2016). Some authors paid great attention to the relationship between self-punishment and guilty, e.g. Alaaeldeen (2003), Ashaboun (2012), and Saleh (2002), while others addressed the relationship between self-compassion and aggressive behavior, such as As-Saeedy (2017). All studies were applied to students, while Almomney and 
Shawashra (2013) covered the residents of the Reformation and Qualification Centers, and Saleh (2002) explored a sample of the patients with psychosomatic disorders. The present study is applied to a sample of students at Umm Al-Qura University and University of Jeddah.

The author benefited from literature in articulating the hypotheses and developing the psychological scales. However, the present study differs from literature because there is not a study, to the author's knowledge, that has covered all these variables.

\section{Hypotheses}

1. There are no statistically significant differences in the means of TTH and healthy students on the psychological stress scale in favor of students with TTH.

2. There are no statistically significant differences in the means of TTH and healthy students on the self-compassion scale in favor of students with TTH.

3. There are no statistically significant differences in the means of TTH and healthy students on the self-punishment scale in favor of students with TTH.

\section{Methodology}

Method: The author adopted the descriptive approach because it is appropriate to the nature and variables of the study.

\subsection{Sampling}

(1) Pilot sample: It covered (100) students aged 18-24 years at the different specializations of Umm Al-Qura University and University of Jeddah to verify the validity, reliability, and appropriateness of the tools.

(2) Main sample: It comprised (235) students at the different specializations and the visitors of the medical clinics at Umm Al-Qura University and University of Jeddah and the visitors of the University Hospital at King Abdulaziz University aged $18-40$ years.

Table 1. Preliminary description of the sample according to the major variables

\begin{tabular}{|c|c|c|c|}
\hline \multicolumn{2}{|c|}{ Description } & \multirow{2}{*}{$\begin{array}{c}\text { Frequency } \\
235\end{array}$} & \multirow{2}{*}{$\begin{array}{c}\text { Percentage } \\
100 \%\end{array}$} \\
\hline Gendor & Females & & \\
\hline 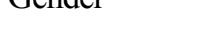 & Total & 235 & $100 \%$ \\
\hline \multirow{3}{*}{$\begin{array}{l}\text { Academic } \\
\text { qualification }\end{array}$} & University & 228 & $97 \%$ \\
\hline & Post-graduate & 7 & $3 \%$ \\
\hline & Total & 235 & $100 \%$ \\
\hline \multirow{7}{*}{ Age } & $18-22$ & 43 & $18.3 \%$ \\
\hline & $22-40$ & 116 & $49.5 \%$ \\
\hline & $20-25$ & 65 & $27.6 \%$ \\
\hline & $26-30$ & 8 & $3.4 \%$ \\
\hline & $31-35$ & 2 & $0.8 \%$ \\
\hline & $36-40$ & 1 & $0.4 \%$ \\
\hline & Total & 235 & $100 \%$ \\
\hline \multirow{3}{*}{ Marital status } & Married & 34 & $13.8 \%$ \\
\hline & Single & 201 & $85.5 \%$ \\
\hline & Total & 235 & $100 \%$ \\
\hline
\end{tabular}

Source: The data was obtained by calculating frequency and percentage

(1) Defining the sample: The higher and lower quartiles of the total sample $(\mathrm{N}=235)$ were calculated on the TTH scale. Based on the total results, the sample was defined, as follows: (59) students with TTH in the higher quartile and (59) healthy students in the lower quartile. Accordingly, the significance of differences was tested to answer the study questions and to validate its hypotheses. 


\subsection{Tools}

a. TTH scale (developed by the author)

By reviewing the psychological Arab literature of scales, the author could not find a relevant scale, but the scale of Shoqer (2003). She utilized it in developing the present scale, theoretical framework, and literature.

The scale comprises (54) items distributed to (3) domains to measure the level of TTH, as follows:

Domain one comprises (15) items on the most significant somatic symptoms and pains of TTH.

Domain two consists of (20) items on the emotional, psychological, behavioral, and social disorders.

Domain three covers (19) items illustrating the personality type of patients with TTH. The scale is answered on a four-point Likert scale (above average- average- below average- very low) marked (3-2-1-0). The scale was rated $(0-162)$, with the high degree indicating severe TTH, the low degree suggesting moderate TTH $(0-108)$, and mild TTH (0-54).

Psychometric features

Validity:

Table 2. Internal validity of the TTH scale

\begin{tabular}{cccccccccccc}
\hline \multicolumn{10}{c}{ Items and correlation coefficients between the item and the total score of the scale } \\
\hline Item & 1 & 2 & 3 & 4 & 5 & 6 & 7 & 8 & 9 & 10 \\
Correlation coefficient & $* * 0.56$ & $* * 0.63$ & $* * 0.52$ & $* * 0.54$ & $* * 0.65$ & $* * 0.64$ & $* * 0.57$ & $* * 0.67$ & $* * 0.61$ & $* * 0.60$ \\
Item & 11 & 12 & 13 & 14 & 15 & 16 & 17 & 18 & 19 & 20 \\
Correlation coefficient & $* 0.47$ & $* * 0.48$ & $* * 0.66$ & $* * 0.68$ & $* * 0.64$ & $* * 0.65$ & $* * 0.63$ & $* * 0.64$ & $* * 0.68$ & $* * 0.68$ \\
Item & 21 & 22 & 23 & 24 & 25 & 26 & 27 & 28 & 29 & 30 \\
Correlation coefficient & $* * 0.66$ & $* * 0.58$ & $* * 0.62$ & $* * 0.62$ & $* * 0.64$ & $* * 0.56$ & $* * 0.54$ & $* * 0.56$ & $* * 0.66$ & $* * 0.57$ \\
Item & 31 & 32 & 33 & 34 & 35 & 36 & 37 & 38 & 39 & 40 \\
Correlation coefficient & $* * 0.57$ & $* * 0.59$ & $* * 0.59$ & $* * 0.64$ & $* * 0.65$ & $* * 0.55$ & $* * 0.54$ & $* * 0.40$ & $* * 0.63$ & $* * 0.51$ \\
Item & 41 & 42 & 43 & 44 & 45 & 46 & 47 & 48 & 49 & 50 \\
Correlation coefficient & $* * 0.44$ & $* * 0.68$ & $* * 0.61$ & $* * 0.42$ & $* * 0.67$ & $* * 0.32$ & $* * 0.20$ & $* * 0.52$ & $* * 0.56$ & $* * 0.59$ \\
Item & 51 & 52 & 53 & 54 & & & & & &
\end{tabular}

$(* *)$ significant at the level of $(0.01)$

(*) significant at the level of (0.05)

Source: The data was obtained by calculating the correlation coefficients between each item and total score

Table 2 shows that the correlation coefficients between the items and the total score of the scale ranged (0.20-0.68).

They were statistically significant at the level of $(0.01)$, suggesting the internal validity of the scale.

Reliability

The reliability of the scale was defined between each domain, the total score and the total scale, as shown in Table (3).

Table 3. Reliability values of the TTH scale and its domains

\begin{tabular}{ccc}
\hline Tools & Alpha & Gutiman \\
\hline The TTH scale & $* * 0.95$ & $* * 0.94$ \\
Somatic symptoms & $* * 0.91$ & $* * 0.89$ \\
Emotional and psychological disorders & $* * 0.93$ & $* * 0.91$ \\
Personality traits & $* * 0.87$ & $* * 0.85$ \\
\hline
\end{tabular}

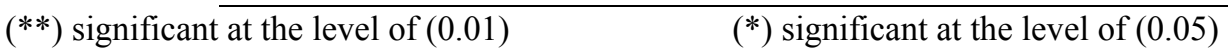

Source: The data was obtained by calculating Alpha and Gutiman for the scale and its domains

Table (3) shows that the reliability coefficients of the total TTH scale and its domains were all significant at the level of (0.01), suggesting its reliability. 
b. Psychological stress scale (developed by the author)

The scale comprises (57) items distributed to seven domains on the stress and coping strategies: (10) items for family and social stress, (8) items for emotional problems, (10) items for cognitive and psychological stress, (8) items for academic stress, (8) items for depression and despair feelings, (5) items for economic stress, and (8) items for health and personal stress. The scale is answered on a four-point Likert scale (above average- average- below average- very low) marked (4-3-2-1). The high degree indicates a high psychological stress of the sub-domain and the low degree suggests a low degree of stressful events.

Psychometric features

Validity

Table 4. Internal validity of the psychological stress scale

\begin{tabular}{cccccccccccc}
\hline \multicolumn{1}{c}{ Items and correlation coefficients between the item and the total score of the scale } \\
\hline Item & 1 & 2 & 3 & 4 & 5 & 6 & 7 & 8 & 9 & 10 \\
Correlation coefficient & $* * 0.43$ & $* * 0.53$ & $* * 0.48$ & $* * 0.35$ & $* * 0.42$ & $* * 0.43$ & $* * 0.55$ & $* * 0.59$ & $* * 0.52$ & $* * 0.44$ \\
Item & 11 & 12 & 13 & 14 & 15 & 16 & 17 & 18 & 19 & 20 \\
Correlation coefficient & $* * 0.57$ & $* * 0.50$ & $* * 0.57$ & $* * 0.49$ & $* * 0.55$ & $* * 0.54$ & $* 0.58$ & $* * 0.27$ & $* * 0.36$ & $* * 0.57$ \\
Item & 21 & 22 & 23 & 24 & 25 & 26 & 27 & 28 & 29 & 30 \\
Correlation coefficient & $* * 0.47$ & $* * 0.55$ & $* * 0.56$ & $* * 0.58$ & $* * 0.65$ & $* * 0.59$ & $* * 0.74$ & $* * 0.58$ & $* * 0.56$ & $* * 0.61$ \\
Item & 31 & 32 & 33 & 34 & 35 & 36 & 37 & 38 & 39 & 40 \\
Correlation coefficient & $* * 0.61$ & $* * 0.56$ & $* * 0.63$ & $* * 0.65$ & $* * 0.60$ & $* * 0.52$ & $* * 0.61$ & $* * 0.54$ & $* * 0.43$ & $* * 0.57$ \\
Item & 41 & 42 & 43 & 44 & 45 & 46 & 47 & 48 & 49 & 50 \\
Correlation coefficient & $* * 0.65$ & $* * 0.43$ & $* * 0.57$ & $* * 0.53$ & $* * 0.50$ & $* * 0.57$ & $* * 0.62$ & $* * 0.50$ & $* * 0.56$ & $* * 0.65$ \\
Item & 51 & 52 & 53 & 54 & 55 & 56 & 57 & & &
\end{tabular}

$(* *)$ significant at the level of $(0.01)$

(*) significant at the level of $(0.05)$

Source: The data was obtained by calculating the correlation coefficients between each item and total score

Table (4) shows that the correlation coefficients between the items and the total score of the scale ranged $(0.36$ :

$0.74)$. They were statistically significant at the level of $(0.01)$, suggesting the internal validity of the scale.

Reliability

The reliability of the scale was defined in various ways, as shown in Table (5).

Table 5. Reliability of the psychological stress scale

\begin{tabular}{cccc} 
& \multicolumn{1}{c}{ Tools } & Alpha coefficient & Gutiman \\
\cline { 2 - 4 } & psychological stress scale & $* * 0.96$ & $* * 0.95$ \\
\hline (*) significant at the level of $(0.01)$ & $(*)$ significant at the level of $(0.05)$
\end{tabular}

Source: The data was obtained by calculating Alpha and Gutiman for the scale and its domains

Table (5) shows that the reliability coefficients (Cronbach alpha and Gutiman) of the psychological stress scale were significant at the level of $(0.01)$, suggesting its reliability.

In addition, the reliability was estimated using test-retest. The reliability was calculated after two applications with an interval of (15) days on a sample of the secondary school and university students, as shown in Table 6. 
Table 6. Reliability coefficients of the psychological stress scale

\begin{tabular}{cccc}
\hline Domains & Retest & Cronbach alpha & Reliability \\
\hline Family and social stress & 0.58 & 0.68 & 0.77 \\
Emotional problems & 0.78 & 0.82 & 0.74 \\
Cognitive and psychological stress & 0.68 & 0.69 & 0.69 \\
Academic stress & 0.88 & 0.80 & 0.79 \\
Economic stress & 0.78 & 0.77 & 0.77 \\
Health and personal stress & 0.82 & 0.81 & 0.74 \\
Depression and despair feelings & 0.65 & 0.68 & 0.77 \\
Total & 0.88 & 0.86 & 0.89
\end{tabular}

Source: The data was obtained by calculating retest and Cronbach alpha

Table 6 shows high values of the reliability coefficients of the domains and the scale of psychological stress, suggesting its high reliability.

c. Self-Compassion scale (developed by Neff, 2003)

The scale comprises (26) items, (3) basic domains, and (6) sub-domains: (5) items for self-kindness, (5) items for self-judgement, (4) items for common humanity, (4) items for isolation, (4) items for mindfulness, and (4) items for over-identification. The scale is answered on a five-point Likert scale (very high- above average- averagebelow average- very low) marked (5-4-3-2-1). The high degree indicates a high degree of compassion of the sub-domain and the low degree suggests a low degree of self-compassion. On handling the sub-domains only, all the items are positive. However, the items (2-4-6) are marked inversely when approaching the total score.

Psychometric features

Validity

Table 7. Internal validity of the self-compassion scale

\begin{tabular}{cccccccccccccccc}
\hline \multicolumn{7}{c}{ Items and correlation coefficients between the item and the total score of the scale } \\
\hline Item & 1 & 2 & 3 & 4 & 5 & 6 & 7 & 8 & 9 & 10 \\
Correlation coefficient & $* * 0.40$ & $* * 0.20$ & $* * 0.62$ & $* * 0.42$ & $* 0.64$ & $* * 0.34$ & $* * 0.61$ & $* * 0.43$ & $* * 0.59$ & $* * 0.67$ \\
Item & 11 & 12 & 13 & 14 & 15 & 16 & 17 & 18 & 19 & 20 \\
Correlation coefficient & $* * 0.41$ & $* * 0.65$ & $* * 0.40$ & $* * 0.68$ & $* * 0.71$ & $* * 0.50$ & $* * 0.63$ & $* * 0.55$ & $* * 0.66$ & $* * 0.47$ \\
Item & 21 & 22 & 23 & 24 & 25 & 26 & & & &
\end{tabular}

$(* *)$ significant at the level of $(0.01) \quad\left(^{*}\right)$ significant at the level of $(0.05)$

Source: The data was obtained by calculating the correlation coefficients between each item and total score

Table 7 shows that the correlation coefficients of the items and the total score ranged $(0.68: 0.52)$. They were significant at the level of (0.01), suggesting its internal validity.

Reliability

The reliability of the scale was defined in various ways (Cronbach alpha and Gutiman), as shown in Table 8 .

Table 8. Reliability of the self-compassion scale

\begin{tabular}{ccc}
\hline Tools & Alpha coefficient & Gutiman \\
\hline Self-compassion scale & $* * 0.83$ & $* * 0.83$ \\
\hline
\end{tabular}

$(* *)$ significant at the level of $\left.(0.01) \quad \quad{ }^{*}\right)$ significant at the level of $(0.05)$

Source: The data was obtained by calculating Alpha and Gutiman for the scale and its domains

Table (8) shows that the reliability coefficients (Cronbach alpha and Gutiman) of the self-compassion scale were significant at the level of (0.01), suggesting its reliability. 
d. Self-Punishment scale (developed by Shoqer, 2006)

Reviewer's validity

Face validity of the tool was verified by showing it to a number of reviewers specialized in psychology to review the clarity of the items and to suggest modifications. They agreed on (90\%) of the items.

Validity

Table 9. Internal validity of the self-punishment scale

\begin{tabular}{cccccccccccc}
\hline \multicolumn{1}{c}{ Items and correlation coefficients between the item and the total score of the scale } \\
\hline Item & 1 & 2 & 3 & 4 & 5 & 6 & 7 & 8 & 9 & 10 \\
Correlation coefficient & $* 0.15$ & $* * 0.19$ & $* * 0.37$ & $* * 0.30$ & $* * 0.25$ & $* * 0.42$ & $* * 0.44$ & $* * 0.42$ & $* * 0.50$ & $* * 0.40$ \\
Item & 11 & 12 & 13 & 14 & 15 & 16 & 17 & 18 & 19 & 20 \\
Correlation coefficient & $* * 0.35$ & $* * 0.47$ & $* * 0.36$ & $* * 0.57$ & $* * 0.47$ & $* * 0.51$ & $* 0.56$ & $* * 0.44$ & $* * 0.33$ & $* * 0.36$ \\
Item & 21 & 22 & 23 & 24 & 25 & 26 & 27 & 28 & 29 & 30 \\
Correlation coefficient & $* * 0.32$ & $* * 0.19$ & $* * 0.58$ & $* * 0.67$ & $* * 0.40$ & $* * 0.58$ & $* * 0.60$ & $* * 0.71$ & $* * 0.56$ & $* * 0.35$ \\
Item & 31 & 32 & 33 & 34 & 35 & 36 & 37 & 38 & 39 & 40 \\
Correlation coefficient & $* * 0.38$ & $* * 0.69$ & $* * 0.55$ & $* * 0.40$ & $* * 0.28$ & $* * 0.57$ & $* * 0.41$ & $* * 0.55$ & $* * 0.31$ & $* * 0.60$ \\
Item & 41 & 42 & 43 & 44 & 45 & 46 & 47 & 48 & 49 & 50 \\
Correlation coefficient & $* * 0.69$ & $* * 0.64$ & $* * 0.54$ & $* * 0.60$ & $* * 0.68$ & $* * 0.58$ & $* * 0.62$ & $* * 0.50$ & $* * 0.53$ & $* * 0.49$ \\
Item & 51 & 52 & & & & & & & &
\end{tabular}

$(* *)$ significant at the level of $(0.01)$

$(*)$ significant at the level of $(0.05)$

Source: The data was obtained by calculating the correlation coefficients between each item and total score

Table 9 shows that the correlation coefficients of the items and the total score ranged $(0.15: 0.71)$. They were significant at the levels of $(0.01 \& 0.05)$, suggesting its internal validity.

Reliability

The reliability of the scale was defined by (Cronbach alpha and Gutiman), as shown in Table (10).

Table 10. Reliability of the self-punishment scale

\begin{tabular}{ccc}
\hline Tools & Alpha coefficient & Gutiman \\
\hline Self-punishment scale & $* * 0.93$ & $* * 0.93$ \\
\hline
\end{tabular}

$(* *)$ significant at the level of $(0.01) \quad \quad(*)$ significant at the level of $(0.05)$

Source: The data was obtained by calculating Alpha and Gutiman for the scale and its domains

Table 10 shows that the reliability coefficients (Cronbach alpha and Gutiman) of the self-punishment scale were significant at the level of (0.01), suggesting its high reliability.

\section{Results}

I. Verifying the first hypothesis

Table 11. Significance of differences in the means of TTH and healthy students on the psychological stress scale

\begin{tabular}{cccccc}
\hline Group & Number & $M$. & $S D$. & $T$-value & Significance \\
\hline Students with TTH & 59 & 131.84 & 25.66 & \multirow{2}{*}{$* * 10.13$} & \multirow{2}{*}{ Significant } \\
Healthy & 59 & 85.67 & 23.79 & & \\
\hline
\end{tabular}

Source: The data was obtained by calculating the mean, standard deviation, T-value, and significance

Table 11 shows that there are statistically significant differences in the means of TTH and healthy students on the scale of psychological stress in favor of students with TTH. 


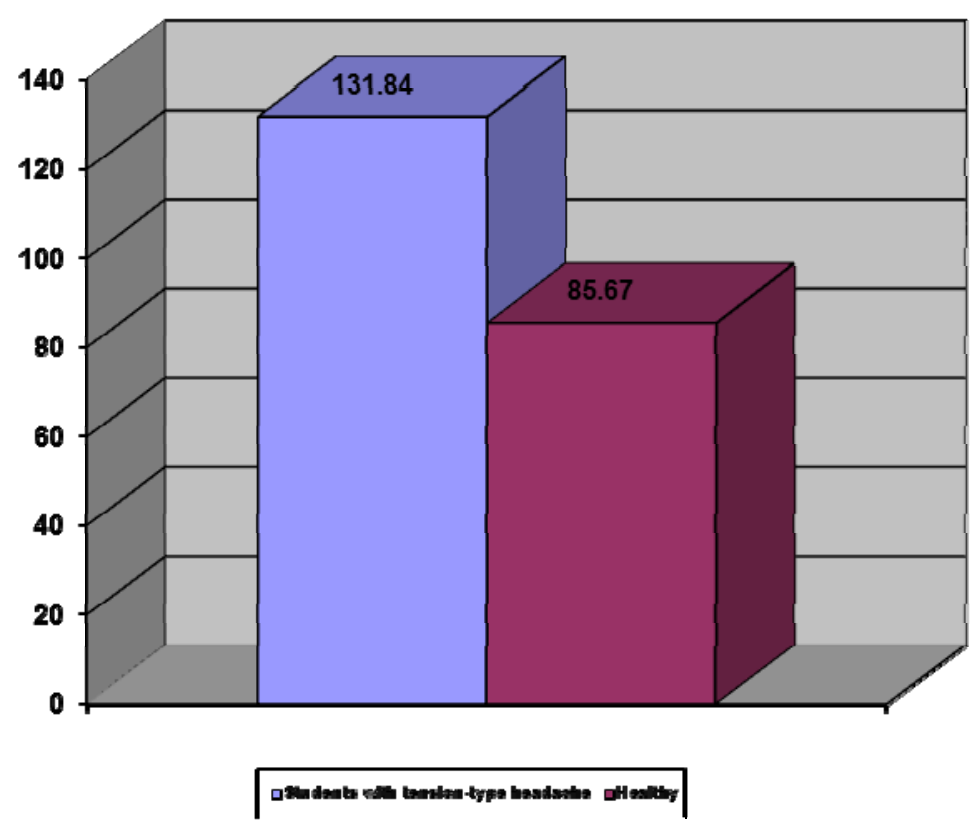

Figure 1. Significance of differences in the means of TTH and healthy students on psychological stress scale

Source: The data was obtained by calculating the mean between TTH and healthy students on the psychological stress scale

There are statistically significant differences in the means of TTH and healthy students on the psychological stress and coping strategies. The T-value was (10.13), which is significant at the level of (0.01). By reviewing the arithmetic means of the groups, they were significant in favor of TTH students. In other words, they suffer more from psychological stress in a statistically significant differences than healthy students.

This result is consistent with the findings of Ficek and Wittrock (1995), reporting that patients with TTH have experienced more stress. It also matches the findings of Puca et al. (1999) and Sarioglu et al. (2003) that patients with TTH suffer from psychological and social stress. It agrees with Leeuw et al. (2005) that (64\%) of the patients with TTH have psychological stress and fatigue because of daily life stress and recurrent headache. As emphasized by Wöber et al. (2006), external factors, e.g. fatigue and psychological stress rating (66.7\%), largely affect headache. Hung et al. (2008) argues that patients with TTH are more sensitive to daily life stressors.

Harboush (2017) reports that many factors affect the individual's health. Thus, one may be prone to TTH. For example, fatigue directly affects the body and TTH recurrently occurs. The life style, in its different forms, can also play a role and have a long-term effect on the health. It includes the type of food, sleep, and problem-solving.

The occurrence of the TTH may result from the inability to confront or cope up with the continuous environmental stress. Furthermore, some works or tasks may cause the occurrence of recurrent stress and tension (Ruby and Ibn Hany, 2018). Abdullah (2016) concludes that the continuous restraint of emotions regarding the stressful events causes tension, anxiety, fear, and anger and negatively affects the internal organs and causes TTH. Alanzey (2007) argues that daily life stress, especially the academic and work stress, may be a psychological trigger of TTH. When reacting to the stressful events physiologically, the autonomic nervous system is activated, causes changes to the hormones, and increases the speed of breath. Accordingly, there are changes to the body structure. Socialization, including life circumstances and social relationships, plays a significant role in the occurrence of TTH.

The result is interpreted that students suffer from TTH due to stress, e.g. academic, family, emotional, and health. This makes them unable to overcome stress and causes recurrent TTH. It suggests that the participants need to acquire many coping strategies to achieve balance, optimism, satisfaction, and mental health. 
II. Verifying the second hypothesis

Table 12. Significance of differences in the means of TTH and healthy students on the self-compassion scale

\begin{tabular}{cccccc}
\hline Group & Number & $M$. & $S D$. & T-value & Significance \\
\hline Students with TTH & 59 & 81.49 & 10.43 & \multirow{2}{*}{$* * 5.14$} & Significant \\
Healthy & 59 & 67.61 & 17.92 & & \\
\hline
\end{tabular}

Source: The data was obtained by calculating the mean, standard deviation, T-value, and significance

Table 12 shows that there are statistically significant differences in the means of TTH and healthy students on the self-compassion scale in favor of students with TTH.
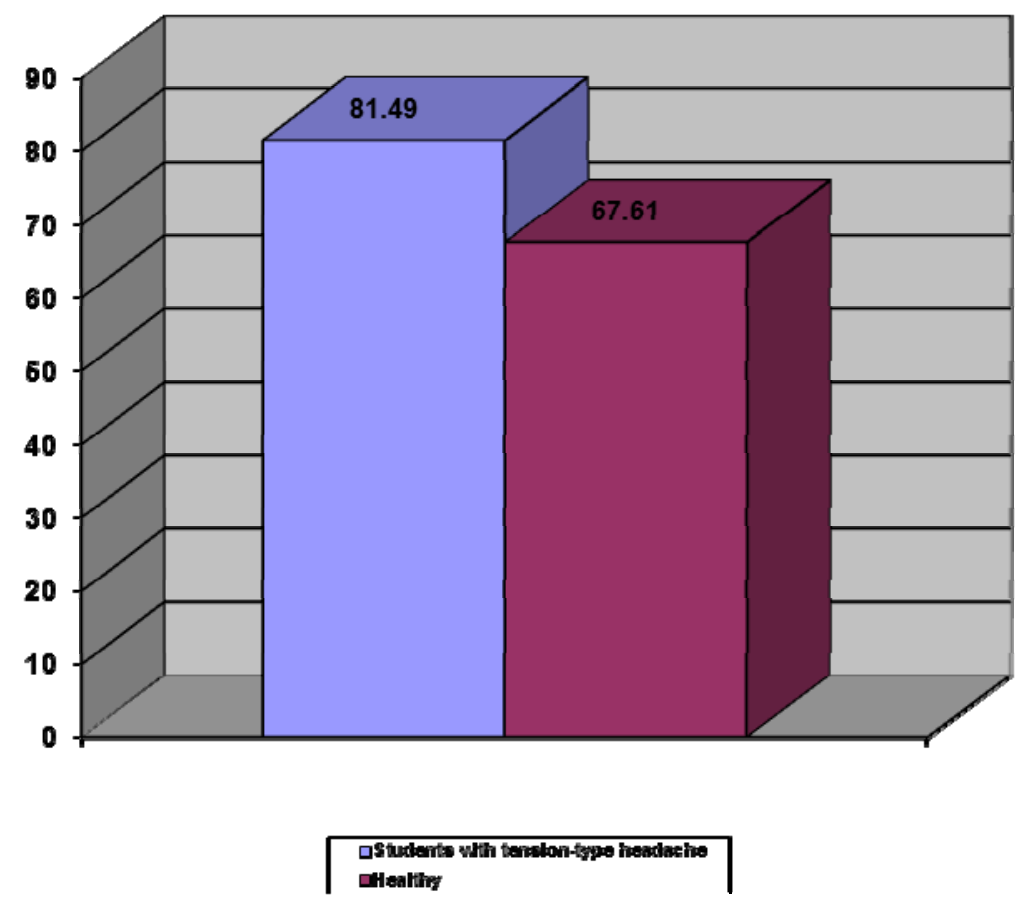

Figure 2. Significance of differences in the means of TTH and healthy students on the self-compassion scale

Source: The data was obtained by calculating the mean between TTH and healthy students on the self-compassion scale

The author argues that this is a logical result for the patients with TTH because of the recurrent events and stress they have. They fail to cope with family, academic, or psychological issues and always criticize themselves. Instead of confrontation, they surrender, fail, and cannot change the way they think. Consequently, they are prone to despair and TTH. Abdurrahman et al. (2015) argued that the self-compassionate in painful and unbalanced situations criticize themselves. The results indicate that university students are not self-compassionate because there are many stress and they are prone to TTH in this stage. Self-compassion is related to the balanced personality and psychological happiness. It enhances and supports their psychological and social adjustment.

The result is consistent with Alasemy (2014) that self-compassion is more than egoism as it means coping with a painful self-experience with high mindfulness. When negative emotions controls the self during pain, self-compassion becomes useless. Olwan (2016) concludes a statistically significant correlation between guilty and aspects of self-compassion and that the aspects of self-compassion help predict guilty. In addition, Alobaedy (2017) argues that university students are self-compassionate and there are no differences in self-compassion among the participants according to gender, specialization, and academic stage. 
III. Verifying the third hypothesis

Table 13. Significance of differences in the means of TTH and healthy students on the self-punishment scale

\begin{tabular}{cccccc}
\hline Group & Number & $M$. & $S D$. & T-value & Significance \\
\hline Students with TTH & 59 & 18.28 & 15.73 & \multirow{2}{*}{$* * 6.56$} & \multirow{2}{*}{ Significant } \\
Healthy & 59 & 4.01 & 5.57 & & \\
\hline
\end{tabular}

Source: The data was obtained by calculating the mean, standard deviation, T-value, and significance

Table 13. shows that there are statistically significant differences in the means of TTH and healthy students on the self-punishment scale in favor of the students with TTH.

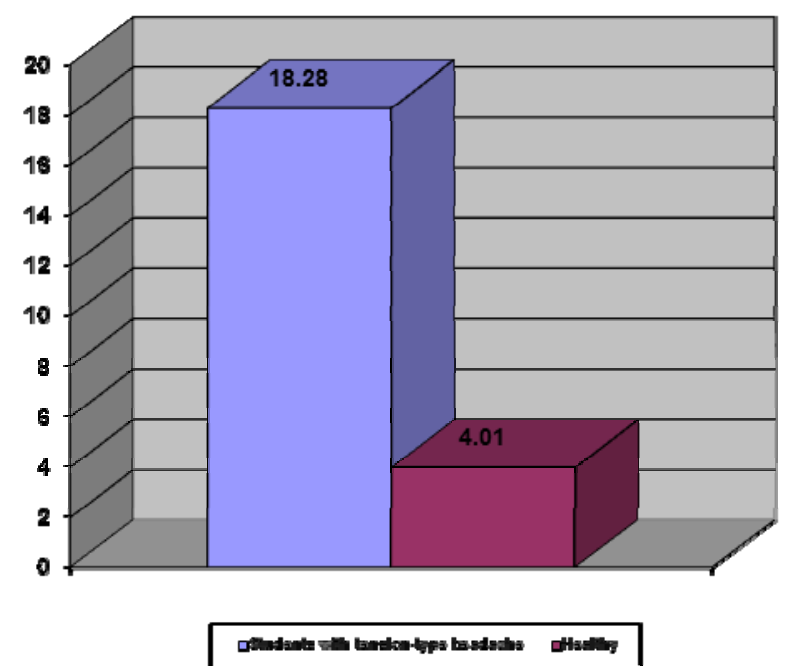

Figure 3. Significance of differences in the means of TTH and healthy students on the self-punishment scale

Source: The data was obtained by calculating the mean between TTH and healthy students on the self-punishment scale

The result is consistent with Saleh (2002) that self-punishment can be a trigger of psychosomatic diseases. Fahmy (1998) illustrates that individual with self-punishment may show a set of attributes, e.g. self-examination, outrage, and continuous physical and emotional punishment in addition to maladjustment and failure (Gazar, 2001). Ongoing family quarrels between parents significantly affect the emotional and social development of the children. The children may feel instable, anxious, and afraid that can affect their self-concept up to self-punishment (Azzaher, 2004).

Self-concept can be affected by social factors, e.g. others' negative perspective, and self-punishment occurs (Alaley, 2003). That is, self-punishment may be due to the social context. The others' perspective and dealing with the individual is a basis for self-concept.

The author interprets the result as natural because of the heavy academic burdens, family stress, and painful events that individuals cannot cope with. Consequently, they feel afraid and anxious. This negatively affects their self-concept and motivates them to have abnormal behaviors. They lose self-control and become prone to the psychosomatic disorders, i.e. TTH.

\section{Recommendations}

1. Considering TTH as a somatic symptom for many psychological diseases.

2. Developing consulting programs to help adolescents alleviate the severity of psychological stress and self-compassion they have, causing various somatic symptoms.

3. Developing consulting programs to help TTH patients reduce headache because it negatively affects their life, future, and social adjustment.

4. Regarding adolescence as a transitional stage between childhood and adulthood that significantly affects the individual's psychological and physical health and adjustment. 


\section{References}

Aaseth, K., Grande, R., Lundqvist, C. \& Russell, M. (2009). Interrelation of chronic tension-type headache with and without medication overuse and migraine in the general population. International Headache Society, 29(3), 331-337. https://doi.org/10.1111/j.1468-2982.2008.01729.x.

Abdullah, M. (2016). Introduction to mental health. Amman: Dar Alfikr.

Abdurrahman, M., Alomary, A., Alasemy, R. \& Aldabaa, F. (2015). Self-compassion scale: A field-study to validate the self-compassion scale among Arab samples. Cairo: Dar Alketab Alhadeeth.

Alaaeldeen, G. (2016). Guilty and self-punishment among the students of the Hashemite University. Journal of Educational Sciences, 11(4), 115-129.

Alanzey, F. (2007). Somatic symptoms inventory and its relation to academic achievement among the students of the Public Authority for Applied Education and Training in Kuwait. Paper presented at the First Regional Conference of Psychology, Egyptian Psychologist Association, Cairo.

Alaley, M. (2003). Self-concept, impact of some demographic features, and its relation to self-immolation among the secondary school teachers in Jenin and Nabuls (Master thesis). College of Graduate Studies, An-Najah National University.

Alasemy, R. (2014). Self-compassion and its relation to some personality traits among King Khalid University students. Damascus University Journal for Educational \& Psychological Sciences, 30(1), 17-56.

Alesawey, A. (2002). Psychosomatic disorders. Lebanon: Dar Elrateb Publishing House.

Alkhaled, M. (2005). The benefit of MRI in the causal diagnosis of chronic headache (Doctoral dissertation). Faculty of Medicine, University of Aleppo.

Alkhateeb, G. (1993). Modifying the behavior of the disabled children: A guidebook to parents and teachers. Amman: Dar Eshraq.

Almenshawy, A. (2016). A causal model of the correlation of self-compassion, fatigue, and academic resilience of the student teacher. Journal of the Faculty of Education in Alexandria-Egypt, 26(5), 153-225.

Almomney, F. \& Shawashra, O. (2013). Self-punishment and its relationship to irrational beliefs among the residents at the Jordanian Reformation and Qualification. The Islamic University Journal of Educational and Psychology Studies, 21(2), 135-159.

Alobaedy, A. 2017. Self-compassion among university students in the light of some variables. Journal of Social Sciences, (26), 41-55.

Ashaboun, D. (2012). Guilty and its relation to anxiety and self-confidence: A field study on the students of grades four and nine of Damascus's public basic schools (Doctoral dissertation). College of Education, Damascus University, Damascus.

Askar, A. (2000). Life stress and treatment styles: Mental and physical health at the time of tension and anxiety $\left(2^{\text {nd }}\right.$ ed.). Cairo: Dar Alketab Alhadeeth.

Assahley, A. (2010). Styles of confronting psychological stress among youth patients at the psychiatric hospital in Medina (Doctoral dissertation). Umm Al-Qura University, Makkah.

Awad, E. (2002). Headache and head pain- Causes, types, and treatment. Cairo: Al-Ahram Center for Translation \& Publishing.

Ashareef, B. (2006). The impact of a collective program of modifying cognitive distortions on reducing depression and improving the level of adjustment among the basic and high school students (Doctoral dissertation). School of Graduate Studies, University of Jordan, Amman.

As-Saeedy, S. (2017). Self-punishment and aggression and their relationship to adolescence. Humanities and Social Sciences, 44(1), 79-90.

Azzaher, Q. (2004). Self-concept: Theory and practice. Jordan: Dar Wael for Publishing.

Bannister, R. (1992). Brain and Bannister's clinical neurology ( ${ }^{\text {th }}$ ed.). Oxford: Oxford University Press.

Beghi, E., Allais, G., Coretelli, P., D'Amico, D., De Simone, R., d'Onofrio, F., Genco, S., Manzoni, C., Moschiano, F., Tonini, M., Torelli, P., Quartaroli, M., Roncolato, M., Salvi, S. \& Bussone, G. (2007). Headache and anxiety-depressive disorder comorbidity. Neurological Sciences, 28(2), 217-219. 
Belqassem, H. (1992). Psychological treatment of chronic tension-type headache: Assessing the effectiveness of two types (Master thesis). University of Algiers.

Carasco, M. \& Kröner-Herwig, B. (2016). Psychological predictors of headache remission in children and adolescents. Adolescent Health, Medicine and Therapeutics, 7, 59-66. https://doi.org/ 10.2147/AHMT.S97925.

Carlsson, J., Larsson, B. \& Mark, A. (1996). Psychosocial functioning in schoolchildren with recurrent headaches. Headache, 36(2), 77-82. https://doi.org/10.1046/j.1526-4610.1996.3602077.x

Crocker, J., \& Canevello, A. (2008). Creating and undermining social support in communal relationships: The role of compassionate and self-image goals. Journal of Personality and Social Psychology, 95(3), 555. https://doi.org/10.1037/0022-3514.95.3.555

Dahmash, A. (2017). Difficulty level of recognition of emotions (Alexithymia): A comparative descriptive study among aggressive and non-aggressive adolescents (Master thesis). Faculty of Humanities and Social Sciences, University of Mohamed Boudiaf, Algeria.

Donaghy, M. (2001). Brain's diseases of the nervous system (1 $1^{\text {th }}$ ed.). Oxford: Oxford University Press.

Dubojska, A., Split, W. \& Rostowskij, A. (1998). Psychological aspects of tension-type headache. Wiadomości Lekarskie, 51(9-10), 404-8.

Elsherbeny, Z., Damanhorey, R. \& Mathana, E. (2018). Pathways to psychology: Concepts, principles, and bases. Riyadh: Al-Shegrey for Publishing \& Information Technology.

Fahmy, M. (1998). Mental health: Studies on the psychology of adjustment. Cairo: International Publishing Company.

Farghaly, A. (1995). Headache and head pain. Cairo: Nahdet Misr Publishing.

Fielding, J., Young, S., Martin, P. \& Waters, A. (2016). Headache symptoms consistent with migraine and tension-type headaches in children with anxiety disorders. Journal of Anxiety Disorders, 49, 67-74. https://doi.org/10.1016/j.janxdis.2016.04.006.

Ficek, S. \& Wittrock, D. (1995). Subjective stress and coping in recurrent tension-type headache. Headache, 35(8), 455-460. https://doi.org/10.1111/j.1526-4610.1995.hed3508455.x.

Fontana, D. (1993). Managing stress (trans. H. Alfaramawey, Abusreaa, R., Abohatab, F.). Cairo: The Anglo Egyptian Bookshop.

Gazar, S. (2001). Self-concept among the psychosomatic patients (Mater thesis). Faculty of Arts, Ain Shams University, Cairo.

Ghanim, M. (1999). How to overcome psychological stress? The latest scientific methods for the treatment of stress. Cairo: Akhbar Alyoum

Hanafy, A. (1992). Encyclopedia of psychotherapy: Handbook of psychological disorders and treatment. Cairo: Madbouly Bookshop.

Harboush, S. (2017). Health and disease from the perspective of health psychology. Tributaries Journal for Studies and Scientific Research in Social and Human Sciences, 1(2), 247-278.

Hung, C., Liu, C. \& Wang, S. (2008). Precipitating or aggravating factors for headache in patients with major depressive disorder. Journal of Psychosomatic Research, 64(2), 231-245. https://doi.org/10.1016/j.jpsychores.2007.08.012

Ismael, B. (2004). Life stress and psychological disorders. Cairo: The Anglo Egyptian Bookshop.

Izmailova, I., Belopasov, V., Kolosova, O. \& Filippov, B. (2002). Clinical and psychophysiological characteristics of headache in childhood. Zh Nevrol Psikhiatr Im S S Korsakova, 102(4), 4-8.

Juang, K., Wang, S., Fun, J., Lu, S. \& Su, T. (2000). Comorbidity of depressive and anxiety disorders in chronic daily headache and its subtypes. Headache, 40(10), 818-823.

Kaufman, D. (1990). Clinical neurology for psychiatrists ( $3^{\text {rd }}$ ed.). London: W.B Saunders Company.

Kleiner-Fisman, G. (2002). Tension headache. Ontario, Canada: Department of Neurology, University of Toronto.

Kumar, A. (2016). Prevalence of tension-type headache among patients of depressive disorder. Pakistan: Jinnah Postgraduate Medical Centre Karachi. 
Lazarus, S., Coyen, C. \& Folkman, S. (1982). Cognition, emotion, and motivation: The doctoring of Humpty-Dumpty. In Neufeld R. (Ed). Psychological stress and psychopathology, 218-239. New York: McGraw-Hill Book Co.

Leeuw, R., Schmidt, J. \& Carlson, R. (2005). Traumatic stressors and post-traumatic stress disorder symptoms in headache patients. Headache, 45(10), 365-374.

Mansour, E. (2016). Psychological resilience, neurosis, self-compassion and emotional styles among the students of Special Education Department. Journal of Educational, Psychological and Environmental Sciences, 4(16), 161-163.

Ruby, M. \& Ibn Hany, M. (2018). Tension-type headache and its relation to decreasing mental health among the secondary school teachers (a field study at some schools of Tiaret). Al-Jamie Journal in Psychological Studies and Educational Sciences, 8(3), 236-266.

Mongini, F., Rota, E., Deregibus, A., Ferrero, L., Migliaretti, G., Cavallo, F., Mongini T. \& Novello A. (2006). Accompanying symptoms and psychiatric comorbidity in migraine and tension-type headache patients. Journal of Psychosomatic Research, 61(4), 447-51.

Murtagh, J. (1995). Tension headache. Melbourne, Australia: Monash University.

Neff, K. (2003). The development and validation of a scale to measure self-compassion. Self and Identity, 2(3), 223-250. https://doi.org/10.1080/15298860309027.

Neff, K., Kirkpatrick, K. \& Rude, S. (2007). Self-compassion and adaptive psychological functioning. Journal of Research in Personality, 41, 139-154.

Neff, K., \& McGehee, P. (2010). Self-compassion and psychological resilience among adolescents and young adults. Self and Identity59(3), 225-240. https://doi.org/10.1080/15298860902979307.

Olwan, E. (2016). Self-compassion and guilty among juvenile offenders at Social Observation House in Abha. International Interdisciplinary Journal of Education, 5(9), 380-401.

Omdal, R., Waterloo, K., Koldingsnes, W., Husby, G., \& Mellgren, S. (2001). Somatic and psychological features of headache in systemic lupus erythematosus. The Journal of Rheumatology, 28(4), 772-779.

Perozzo, P., Savi, L., Castelli, L., Valfrè, W., Lo Giudice, R., Gentile, S., Rainero, I. \& Pinessi, L. (2005). Anger and emotional distress in patients with migraine and tension-type headache. Headache, 6(5), 392-399. https://doi.org/10.1007/s10194-005-0240-8.

Rasmussen, B. (2001). Epidemiology of headache. International Headache Society, 21(7), 774-777. https://doi.org/10.1177/033310240102100708.

Puca, F., Genco, S., Prodenzano, M., Sevares, M., Bussone, G., D'Amico, D., Cerbo, R. \& Gala, C., (1999). Psychiatric comorbidity and psychosocial stress in patients with tension-type headache from headache centers in Italy, the Italian Collaborative group for the study of psychopathological factors in primary Headaches. Cephalagia, 19(3), 159-164. https://doi.org/10.1046/j.1468-2982.1999.1903159.x

Qlandar, S. and Abbas, A. (2007). Emotional suppression and relationship to psychological stress among university students. Center of Psychological Research, 26, 1927-1060.

Saema, A. (2018). Guilt and its relation to psychological resilience among the patients at intensive care departments in Gaza Strip's public hospitals (Master thesis). Faculty of Education, Islamic UniversityGaza

Saleh, Q. (2002). Guilty and its relation to psychosomatic diseases: A field study. Journal of the Faculty of Arts, 61(1), 72-98.

Sarioglu, B., Erhan, E., Serdaroglu, G., Doering, B., Erermis, S. \& Tutuncuo, S. (2003). Tension-type headache in children: a clinical evaluation. Pediatrics International, 45(2), 186-189. https://doi.org/10.1046/j.1442-200X.2003.01678.x

Saudi Ophthalmological Society. (2017). Headache: Health education. Retrieved from http://www.sos.org.sa.

Shama, G. (1997). Short notes on psychiatry. Cairo: Dar El Ketab.

Shoqer, Z. (2002). Psychosomatic diseases. Cairo: Nahdet Misr Publishing.

Shoqer, Z. (2003). Scale of the (psychological) tension-type headache: Psychosomatic disorders- a guide. Cairo: Nahdet Misr Publishing. 
Shoqer, Z. (2005). Health disorders-psychosomatic and somatic psychological: 7. Cairo: Nahdet Misr Publishing.

Shoqer, Z. (2006). Scale of diagnosing self-punishment ( $I^{\text {st }}$ ed.). Cairo: Nahdet Misr Publishing.

Shoqer, Z. (2008). Scale of stressful life events in the Arab environment (Egyptian- Saudi) - a guide (4 $4^{\text {th }}$ ed.). Cairo: Nahdet Misr Publishing.

Shoqer, Z. (2009). Positive contribution of psychology to improving the quality of life and reducing future anxiety in a case with (psychological) tension-type headache through online chatting. Proceedings of the Sixth Conference of Psychology at the Faculty of Arts- Tanta University.

Simić, S., Slankamenac, P., Kopitović, A., Jovin, Z. \& Banić-Horvat, S. (2008). Quality of life research in patients suffering from tension type headache. Medicinski pregled, 61(5-6), 215-221.

Söderlund, A. \& Lagerlöf, H. (2016). An individually tailored behavioral medicine treatment in physical therapy for tension-type headache - two experimental case studies. Journal of Pain Research, 9, 1089-1099. https://doi.org/ 10.2147/JPR.S121609.

Stronks, D., Tulen, J., Pepplinkhuizen, I., Verheij, R., Mantel, G., Spinhoven, P. \& Passchier, J. (1999). Personality traits and psychological reactions to mental stress of male migraine patients. Cephalalgia, 19(6), 566- 574. https://doi.org/10.1046/j.1468-2982.1999.019006566.x.

Torelli, P., Abrignani, G., Castellini, P., Lambru, G., \& Manzoni, G. (2008). Human psyche and headache: Tension-type headache. Neurological Sciences, 29(1), 93-5. https://doi.org/10.1007/s10072-008-0896-3.

Venable, V., Carlson, C. \& Wilson, J. (2001). The role of anger and depression in recurrent headache. Headache, 4l(1), 21-30. https://doi.org/10.1046/j.1526-4610.2001.111006021.x.

Waldie, K. \& Poulton, R. (2002). Physical and psychological correlates of primary headache in young adulthood: A 26 year longitudinal study. Journal of Neurology, Neurosurgery, and Psychiatry, 72(1), 86-92.

Wöber, C., Holzhammer, J., Zeitlhoser, J., Wessely, P. \& Wober-Bingol, C. (2006). Trigger factors of migraine and tension-type headache. Headache, 7(4), 188-95. https://doi.org/10.1007/s10194-006-0305-3.

Ying, Y. (2009). Self-compassion to competence and mental health among social work student. Journal of Social Work Education, 45(2), 309-325.

\section{Copyrights}

Copyright for this article is retained by the author(s), with first publication rights granted to the journal.

This is an open-access article distributed under the terms and conditions of the Creative Commons Attribution license (http://creativecommons.org/licenses/by/4.0/). 